\title{
High myopia-sensorineural deafness syndrome
}

INSERM

\section{Source}

INSERM. (1999). Orphanet: an online rare disease and orphan drug data base. High myopia-sensorineural deafness syndrome. ORPHA:363396

High myopia-sensorineural deafness syndrome is a rare genetic disease characterized by high myopia, typically rang ing from -6.0 to -11.0 diopters, and moderate to profound, bilateral, progressive sensorineural hearing loss with prelingual-onset. Affected individuals do not present other systemic, ocular or connective tissue manifestations. 